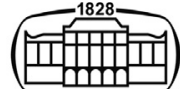

AKADÉMIAI KIADÓ

\section{Acta Veterinaria Hungarica}

$69(2021) 3,211-215$

DOI:

$10.1556 / 004.2021 .00036$

(C) 2021 The Author(s)

\section{RESEARCH ARTICLE}

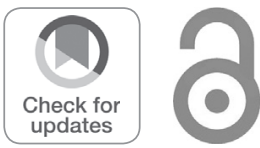

\title{
Occurrence of Escherichia coli producing extended spectrum $\beta$-lactamases in food-producing animals
}

\author{
BENCE BALÁZS $S^{1,2 *} \odot$, JÓZSEF BÁLINT NAGY ${ }^{1,2}$, \\ ZOLTÁN TÓTH ${ }^{1,2}$, FRUZSINA NAGY ${ }^{1,2}$, SÁNDOR KÁROLYI ${ }^{1}$, \\ IBOLYA TURCSÁNYI ${ }^{3}$, ANDREA BISTYÁK ${ }^{3}$, \\ ATTILA KÁLMÁN ${ }^{4}$, RITA SÁRKÖZI ${ }^{5}$ and GÁBOR KARDOS ${ }^{1}$
}

\author{
${ }^{1}$ Department of Medical Microbiology, Faculty of Medicine, University of Debrecen, Nagyerdei krt. \\ 98, Debrecen, H-4032, Hungary \\ ${ }^{2}$ Doctoral School of Pharmaceutical Sciences, University of Debrecen, Debrecen, Hungary \\ ${ }^{3}$ Debrecen Laboratory, Veterinary Diagnostic Directorate, National Food Chain Safety Office, \\ Debrecen, Hungary \\ ${ }^{4}$ Hajdúszoboszló, HAGE Hajdúsági Agráripari Zrt., Hungary \\ ${ }^{5}$ Private Veterinarian
}

Received: 30 June 2020 • Accepted: 2 September 2021

Published online: 21 September 2021

\begin{abstract}
Multidrug resistance due to the production of extended-spectrum beta-lactamases (ESBLs) is a major problem in human as well as in veterinary medicine. These strains appear in animal and human microbiomes and can be the source of infection both in animal and in human healthcare, in accordance with the One Health theorem. In this study we examined the prevalence of ESBL-producing bacteria in food-producing animals. We collected 100 porcine and 114 poultry samples to examine the prevalence of ESBL producers. Isolates were identified using the MALDI-TOF system and their antibiotic susceptibility was tested using the disk diffusion method. ESBL gene families and phylogroups were detected by polymerase chain reactions. The prevalence of ESBL producers was relatively high in both sample groups: $72(72.0 \%)$ porcine and $39(34.2 \%)$ poultry isolates were ESBL producers. Escherichia coli isolates were chosen for further investigations. The most common ESBL gene was CTX-M-1 (79.3\%). Most of the isolates belong to the commensal E. coli phylogroups. The porcine isolates could be divided into three phylogroups, while the distribution of the poultry isolates was more varied. In summary, ESBL-producing bacteria are prevalent in the faecal samples of the examined food-producing animals, with a dominance of the CTX-M-1 group enzymes and commensal E. coli phylogroups.
\end{abstract}

\section{KEYWORDS}

ESBL producers, poultry, swine, asymptomatic carriage

\section{INTRODUCTION}

Bacteria producing extended spectrum $\beta$-lactamases (ESBLs) cause serious problem in human and veterinary healthcare, as these bacteria are resistant to penicillins, broad-spectrum

${ }^{*}$ Corresponding author. Tel.: +3652 255 425. Email: balazs.bence@med. unideb.hu cephalosporins and monobactams. Beta-lactam antibiotics are used frequently in human and veterinary medicine against infections caused by Enterobacterales (Nüesch-Inderbinen et al., 2016). Correlation between the consumption of 3rd and 4th generation cephalosporins and cephalosporin resistance of Escherichia coli was reported in human medicine and in foodproducing animals by the European Centre for Disease Prevention and Control (ECDC), the European Food Safety Authority (EFSA) and the European Medicines Agency (EMA) in a 
joint report (ECDC, EFSA and EMA, 2017). In this manner, ESBL enzymes, found frequently in the background of resistance to multiple beta-lactam antibiotics, became ubiquitous in Enterobacterales, especially in E. coli, spreading on mobile genetic elements and occurring frequently in the gut microbiome of humans or animals. The microbiome thus serves as a reservoir of ESBL producers and ESBL genes, and the human and animal microbiomes are interconnected through the food chain (Silva et al., 2019). Recognising the importance of this relationship, the World Health Organization (WHO), the World Organisation for Animal Health (OIE) and the Food and Agriculture Organization (FAO) established the One-Health concept 'to achieve optimal health and well-being outcomes recognising the interconnections between people, animals, plants and their shared environment' (https://www.onehealthcommission.org/ en/why_one_health/what_is_one_health/).

The aim of this study was to investigate the occurrence of ESBL producers in food-producing animals, and to determine their human pathogenic potential through phylogroup analysis. We also compared these isolates to contemporary isolates from asymptomatic human carriage.

\section{MATERIALS AND METHODS}

\section{Samples and culturing}

A total of 100 porcine and 114 poultry faecal samples were investigated. The samples were cultured on eosin-methylene blue agar supplemented with $2 \mathrm{mg} \mathrm{L}^{-1}$ cefotaxime. This culturing method helped screen the ESBL strains. The antibiogram (antibiotic susceptibility testing) was determined using the Kirby-Bauer disk diffusion method based on the European Committee on Antimicrobial Susceptibility Testing (EUCAST) recommendations against carbapenems (ertapenem, meropenem, imipenem), amoxicillin/clavulanic acid, cefotaxime, cefepime, ciprofloxacin, sulphamethoxazole/trimethoprim (Sumetrolim, SXT) amikacin, tobramycin, and gentamicin; colistin susceptibility was tested by microdilution (Merlin GmBH, Berlin, Germany). The identification of bacterial colonies was performed using Matrix-Assisted Laser Desorption/Ionization Time-of Flight (MALDI-TOF) Biotyper (Bruker Daltonics, Bremen, Germany). The ESBL phenotype was examined using the double-disk synergy test, the cefotaxime and amoxicillin/ clavulanic acid disks placed at $30 \mathrm{~mm}$ apart. The double-disk synergy test was positive when the inhibition zone was enhanced between the two disks (synergy between cefotaxime and the lactamase-inhibitor clavulanic acid). Animalderived isolates were compared to 17 contemporary human isolates originating from asymptomatic faecal or oral carriage collected at the University of Debrecen.

\section{Resistance genes and phylogenetic analysis}

The $b l a_{T E M}, b l a_{C T X-M}$ and $b l a_{S H V}$ genes were detected by PCR, as described previously (Ebrahimi et al., 2014). The bla $a_{C T X-M}$ genes in the CTX-M-1 group were sequenced using group-specific PCR primers. Sequences were analysed by means of CLC DNA Workbench v4.0 (CLC Bio, Aarhus, Denmark).

The phylogenetic analysis of E. coli isolates was performed by the multiplex PCR method of Clermont et al. (2013). This method defines eight phylogroups: phylogroups A1, B1, C, $\mathrm{E}, \mathrm{F}$ and clade I contain mainly commensal strains, while phylogroups B2 and D are responsible for extraintestinal infections in humans. The pandemic ST131 clone and its subclades C1-M27 and the C2 carrying bla $a_{C T X-M-15}$ were detected using the method of Matsumura et al. (2017).

\section{RESULTS}

\section{Prevalence of ESBL producers}

The prevalence of ESBL-producing isolates was markedly different between the porcine and poultry samples, 72 $(72.0 \%)$ and 39 (34.2\%) ESBL-producing isolates were found, respectively. All poultry and 43 porcine isolates were E. coli; 21 Proteus spp., 7 Myroides odoratimimus, 5 Citrobacter freundii, 2 Morganella morganii and 1 Providencia rettgeri were also found among the porcine isolates. Only $E$. coli isolates were investigated further.

\section{Phenotypic resistance}

The phenotypic resistance was similar among the porcine and the poultry E. coli isolates: colistin ( $0.0 \%$ vs. $2.6 \%)$, amikacin (39.5\% vs. $35.9 \%)$, tobramycin (39.5\% vs. $35.9 \%)$, and all E. coli isolates were resistant to cefotaxime and cefepime. In the case of gentamicin, the resistance was higher within porcine isolates $(79.1 \%$ vs. $12.8 \%)$. The SXT resistance showed similar results $(81.4 \%$ vs. $35.9 \%)$. All of the poultry isolates were resistant to ciprofloxacin, while $68.9 \%(30 / 43)$ of the porcine E. coli were resistant to it. All isolates were susceptible to carbapenems (ertapenem, meropenem, imipenem), the drug group of first choice against ESBL-producers in human medicine (Fig. 1).

\section{Prevalence of ESBL gene families}

The distribution of ESBL gene families differed between the two groups of isolates. Group CTX-M-1 was the most common; its prevalence was higher in porcine than in poultry isolates $(39 / 43,90.7 \%$ and $27 / 39,69.7 \%$, respectively). All but one porcine isolates and all poultry isolates carried the $b l a_{C T X-M-1}$ gene, the remaining one porcine isolate harboured $b l a_{C T X-M-3}$; the $b l a_{C T X-M-15}$ gene was not found in the animal isolates. The CTX-M-2 gene family was found only in porcine isolates $(4.7 \%)$, while the CTX-M-9 was found in poultry E. coli (7.7\%). The CTX-M-8 family was totally absent (Fig. 2). The genes of these groups were not sequenced.

\section{Prevalence of $E$. coli phylogroups}

The occurrence of E. coli phylogroups was different in the two types of isolates. Most of the isolates belonged 


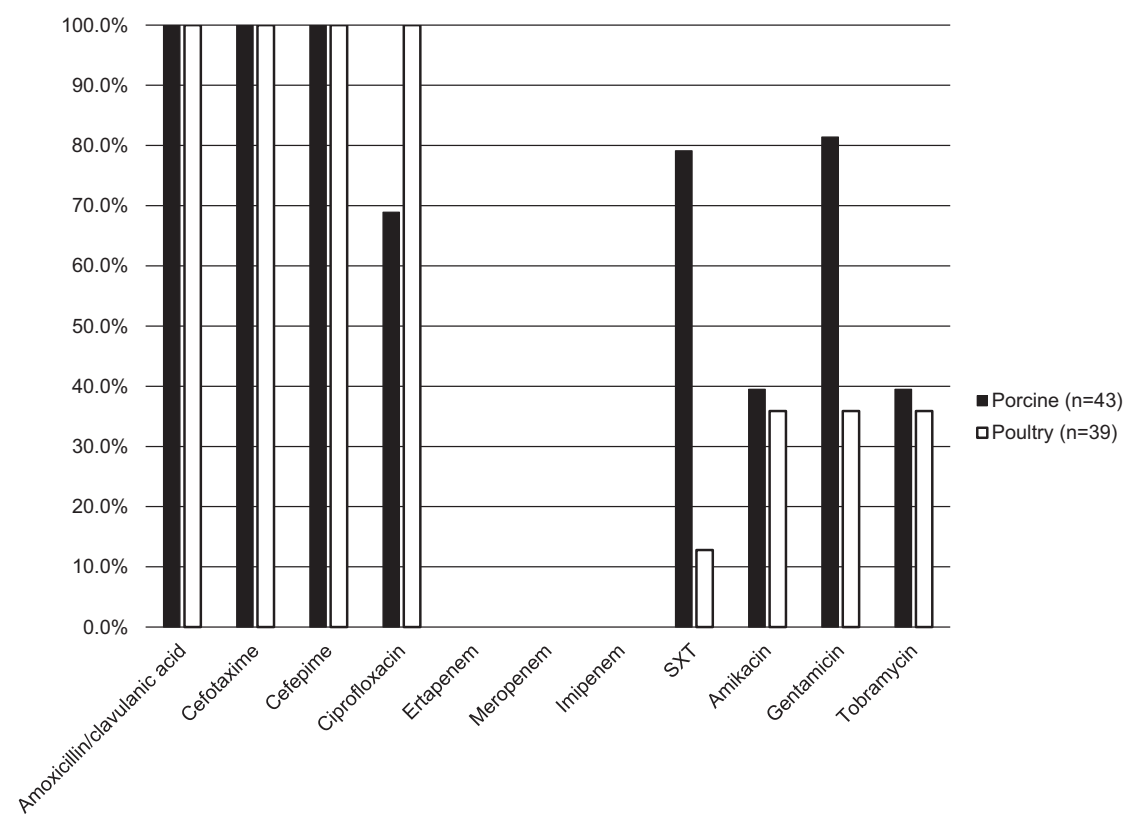

Fig. 1. Proportion of isolates resistant to different antibiotics (SXT $=$ sulphamethoxazole/trimethoprim)

to commensal phylogroups, but the distribution was different, the porcine isolates could be distributed into three phylogroups, while the poultry isolates were more varied, and only phylogroup $\mathrm{D}$ was absent from both types of samples (Fig. 3). Of the porcine isolates, $67.4 \%(29 /$ 43) belonged to phylogroup $C$, while $11.6 \%$ (5/43) and $14.0 \%(6 / 43)$ of these isolates were members of phylogroups $\mathrm{A}$ and $\mathrm{B} 1$, respectively. Most of the poultry isolates belonged to phylogroups $\mathrm{B} 1$ and $\mathrm{E}(30.8 \%$ and $30.8 \%, 12 / 39$, respectively), while $7.7 \%(3 / 39)$ and $12.8 \%(5 / 39)$ of them could be classified into two other commensal phylogroups, $\mathrm{C}$ and F (Fig. 3).

\section{Human isolates}

The antibiotic resistance of human isolates was higher against all examined antibiotics. SXT and aminoglycoside resistance rates were $16 / 17$ and 14/17, respectively; one human isolate was resistant against ertapenem.

The most common ESBL gene was the CTX-M-15 (11/ 17); as expected, four of the isolates proved to be of the clone ST131 clade C2. A single isolate carried blactxm1, another carried CTX-M27 and belonged to the clade ST131/C1-M27. The occurrence of the TEM and SHV gene families was similar among the human isolates (5/17).

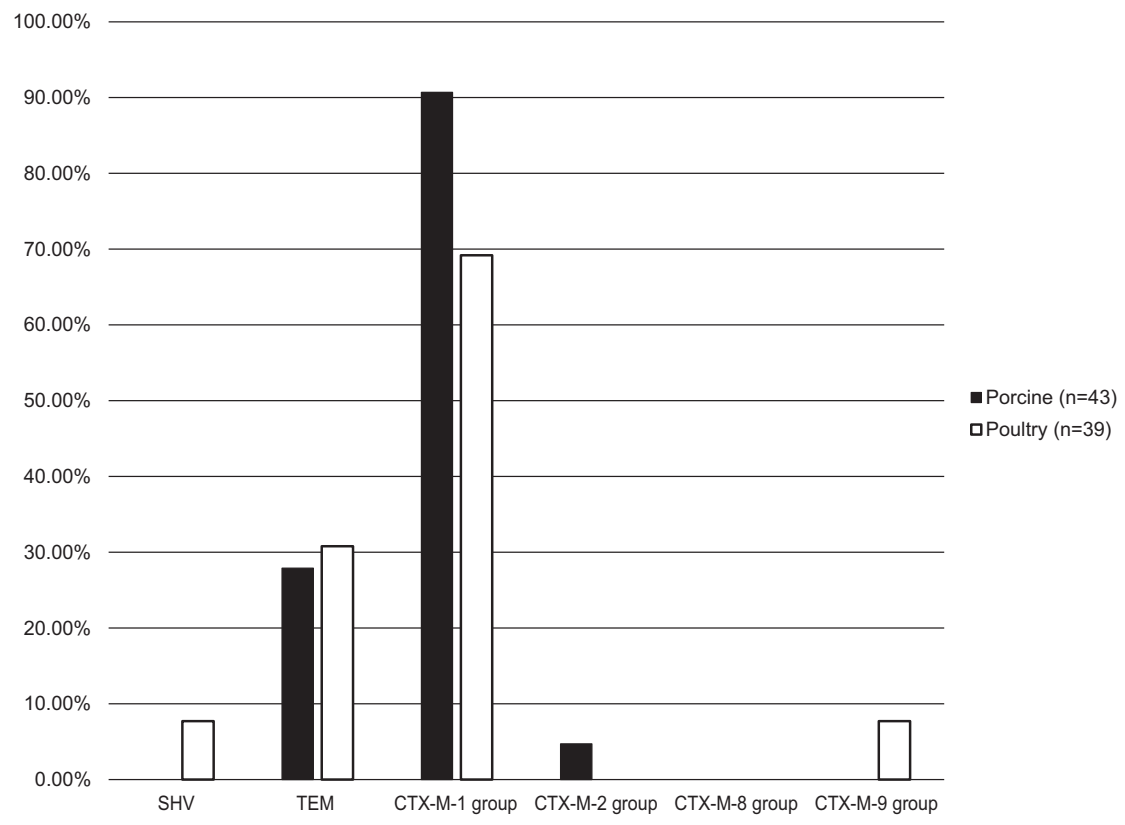

Fig. 2. Result of the PCR examination of ESBL gene families - comparison of porcine and poultry E. coli isolates 


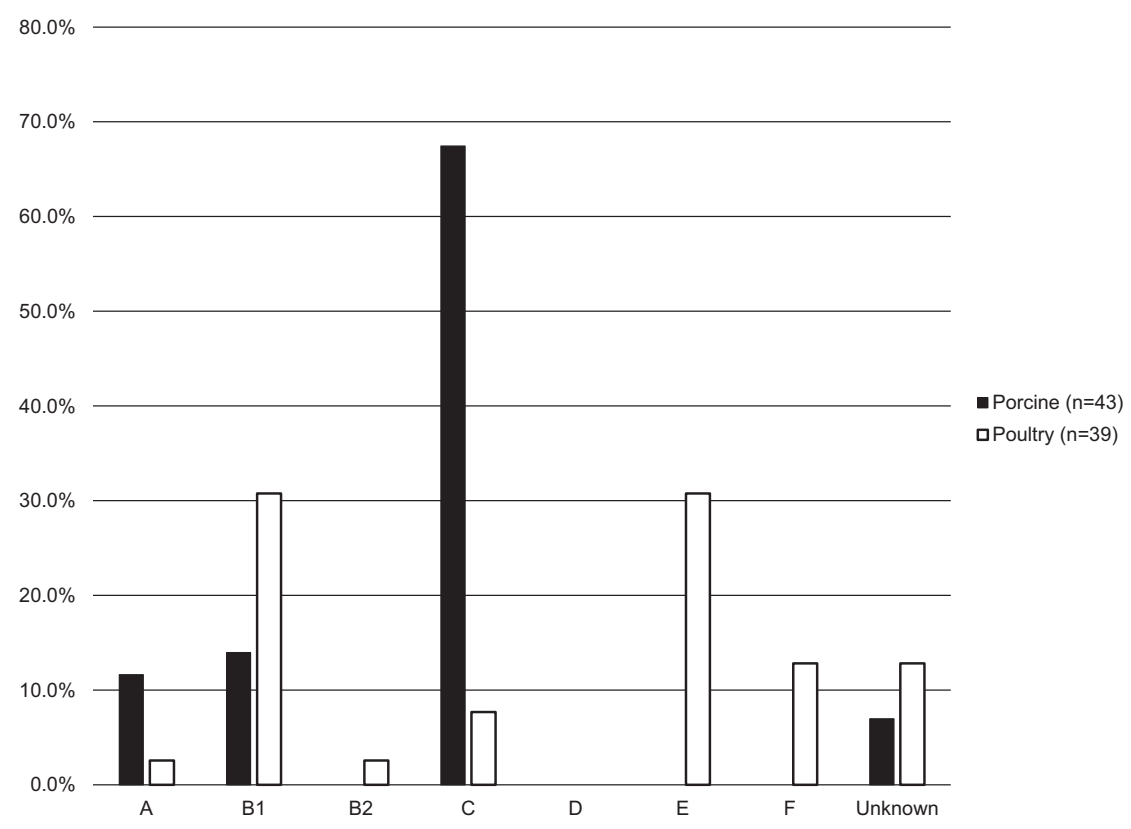

Fig. 3. Result of E. coli extended phylogroup multiplex PCR based on the method of Clermont et al. (2013)

Most isolates belonged to the B2 and D phylogroups (10/17, 58.9\% and 4/17, 23.5\%, respectively); five isolates from the B2 group were of sequence type 131. The remaining isolates belonged to the $\mathrm{A}$ and $\mathrm{E}$ phylogroups.

\section{DISCUSSION}

A high prevalence of ESBL-producing bacteria was found among the poultry and pork isolates. Although the prevalence of ESBL producers was different, the gene distribution was highly similar. These gene patterns did not differ from data of the literature; the vast majority of animal isolates carried $b l a_{C T X-M-1}$ among both types of animal isolates, while in the human isolates bla $a_{C T X-M-15}$ dominated (Bush, 2013; Abraham et al., 2018). This is in line with a previous Hungarian study surveying the faecal carriage of ESBL producers in animals (Tóth et al., 2013) and reporting bla $a_{C T X-M-1}$ as the most common gene.

Very similar results have been reported worldwide, with the dominance of $b l a_{C T X-M-1}$ in poultry (Girlich et al., 2007; Saliu et al., 2017; Gundran et al., 2019), which is horizontally transmitted among different strains (Zurfluh et al., 2014) and may reach humans through the food chain (de Been et al., 2014). Similar dominance and transmission were reported in the case of porcine samples (Abraham et al., 2018). Interestingly, among pig slaughterhouse workers, direct animal contact was associated with a higher risk of carrying ESBL producers (Dohmen et al., 2017), pointing out the importance of transmission through the pork food chain.

Human isolates contemporary with those investigated by Tóth et al. (2013), derived from asymptomatic individuals including animal keepers and abattoir workers, showed a marked dominance of $b l a_{C T X-M-1}$ (Ebrahimi et al., 2014), the other frequent gene was $b l a_{C T X-M-15}$. This proportion shifted towards dominance of the pandemic bla $a_{C T X-M-15}$ carrying the ST131 clone (Ebrahimi et al., 2016a, 2016b), which was also detected in human isolates but, importantly, not in animal isolates in this study.

In general, commensal phylogroups were frequently found among ESBL carriers in animals. In poultry, the phylogroups B2 and D containing extraintestinal pathogens were rare, in line with earlier results (Saliu et al., 2017; op. cit.). In contrast, among porcine isolates the dominant phylogroups among ESBL producers were those associated with intestinal (C) and extraintestinal (A and B1) infections, in the same way as among human isolates (B2 and D).

In animal-derived isolates, $b l a_{C T X-M-1}$ has been the dominant gene in ESBL-producing $E$. coli for more than a decade, which is also detected continuously, but only in a relatively small proportion of human isolates. In contrast, the gene most important in human isolates, $b l A_{C T X-M-15}$ is found exclusively in human isolates and has failed to cross the species barrier. This suggests that the gene flow is continuous between humans and animal isolates but the proportion of shared genes or isolates is small and unimportant in terms of public health epidemiology in Hungary.

\section{ACKNOWLEDGEMENTS}

The authors thank Ákos Tóth for the critical review of the manuscript. Funding: Bence Balázs, Zoltán Tóth and Fuzsina Nagy were supported by ÚNKP-9/3-I (New National Excellence Program of the Ministry for Innovation and Technology) and the EFOP-3.6.3-VEKOP-16-2017-00009 project co-financed by the EU and the European Social Fund. 


\section{REFERENCES}

Abraham, S., Kirkwood, R. N., Laird, T., Saputra, S., Mitchell, T., Singh, M., Linn, B., Abraham, R. J., Pang, S., Gordon, D., M., Trott, D. J. and O'Dea, M. (2018): Dissemination and persistence of extended-spectrum cephalosporin-resistance encoding IncI1-bla CTXM-1 plasmid among Escherichia coli in pigs. ISME J. 12, 2352-2362.

Bush, K. (2013): Proliferation and significance of clinically relevant $\beta$-lactamases. Ann. N. Y. Acad. Sci. 1277, 84-90.

Clermont, O., Christenson, J. K., Denamur, E. and Gordon, D. M. (2013): The Clermont Escherichia coli phylo-typing method revisited: improvement of specificity and detection of new phylo-groups. Environ. Microbiol. Rep. 5, 58-65.

de Been, M., Lanza, V. F., de Toro, M., Scharringa, J., Dohmen, W., Du, Y., Hu, J., Lei, Y., Li, N., Tooming-Klunderud, A., Heederik, D. J., Fluit, A. C., Bonten, M. J. M., Willems, R. J. L., de la Cruz, F. and van Schaik, W. (2014): Dissemination of cephalosporin resistance genes between Escherichia coli strains from farm animals and humans by specific plasmid lineages. PLoS Genet. 10, e1004776.

Dohmen, W., Van Gompel, L., Schmitt, H., Liakopoulos, A., Heres, L., Urlings, B. A., Mevius, D., Bonten, M. J. M. and Heederik, D. J. J. (2017): ESBL carriage in pig slaughterhouse workers is associated with occupational exposure. Epidem. Infect. 145, 2003-2010.

Ebrahimi, F., Mózes, J., Mészáros, J., Juhász, Á. and Kardos, G. (2014): Carriage rates and characteristics of Enterobacteriaceae producing extended-spectrum beta-lactamases in healthy individuals: comparison of applicants for long-term care and individuals screened for employment purposes. Chemotherapy 60, 239-249.

Ebrahimi, F., Mózes, J., Mészáros, J., Juhász, Á., Majoros, L., Szarka, K. and Kardos, G. (2016a): Asymptomatic faecal carriage of ESBL producing Enterobacteriaceae in Hungarian healthy individuals and in long-term care applicants: A shift towards CTX-M producers in the community. Infect. Dis. 48, 557-559.

Ebrahimi, F., Mózes, J., Monostori, J., Gorácz, O., Fésűs, A., Majoros, L., Szarka, K. and Kardos, G. (2016b): Comparison of rates of fecal colonization with extended-spectrum beta-lactamase-producing enterobacteria among patients in different wards, outpatients and medical students. Microbiol. Immunol. 60, 285-294.

ECDC (European Centre for Disease Prevention and Control), EFSA (European Food Safety Authority), and EMA (European
Medicines Agency) (2017): ECDC/EFSA/EMA second joint report on the integrated analysis of the consumption of antimicrobial agents and occurrence of antimicrobial resistance in bacteria from humans and food-producing animals - Joint Interagency Antimicrobial Consumption and Resistance Analysis (JIACRA) Report. EFSA J. 15(4872), 135.

Girlich, D., Poirel, L., Carattoli, A., Kempf, I., Lartigue, M. F., Bertini, A. and Nordmann, P. (2007): Extended-spectrum $\beta$-lactamase CTX-M-1 in Escherichia coli isolates from healthy poultry in France. Appl. Environ. Microbiol. 73, 4681-4685.

Gundran, R. S., Cardenio, P. A., Villanueva, M. A., Sison, F. B., Benigno, C. C., Kreausukon, K., Pichpol, D. and Punyapornwithaya, V. (2019): Prevalence and distribution of bla CTX-M, bla SHV, bla TEM genes in extended-spectrum $\beta$-lactamase-producing E. coli isolates from broiler farms in the Philippines. BMC Vet. Res. 15, 227.

Matsumura, Y., Pitout, J. D., Peirano, G., DeVinney, R., Noguchi, T., Yamamoto, M., Gomi, R., Matsuda, T., Nakano., S., Nagao, M., Tanaka, M. and Ichiyama, S. (2017): Rapid identification of different Escherichia coli sequence type 131 clades. Antimicrob. Agents Chemother. 61, e00179-17.

Nüesch-Inderbinen, M. and Stephan, R. (2016): Epidemiology of extended-spectrum $\beta$-lactamase-producing Escherichia coli in the human-livestock environment. Curr. Clin. Microbiol. Rep. 3, 1-9.

Saliu, E., Vahjen, W. and Zentek, J. (2017): Types and prevalence of extended-spectrum beta-lactamase producing Enterobacteriaceae in poultry. Anim. Health Res. Rev. 18, 46-57.

Silva, N., Carvalho, I., Currie, C., Sousa, M., Igrejas, G. and Poeta, P. (2019): Extended-spectrum- $\beta$-lactamase and carbapenemaseproducing Enterobacteriaceae in food-producing animals in Europe: An impact on public health? In: Capelo-Martínez, J.-L. and Igrejas, G. (eds) Antibiotic Drug Resistance. Chapter 12. John Wiley and Sons, Inc. pp. 261-273.

Tóth, Á., Juhász-Kaszanyitzky, É., Mag, T., Hajbel-Vékony, G., Pászti, J. and Damjanova, I. (2013): Characterization of extended-spectrum $\beta$-lactamase (ESBL) producing Escherichia coli strains isolated from animal and human clinical samples in Hungary in 2006-2007. Acta Microbiol. Immunol. Hung. 60, 175-185.

Zurfluh, K., Wang, J., Klumpp, J., Nüesch-Inderbinen, M., Fanning, S. and Stephan, R. (2014): Vertical transmission of highly similar blaCTX-M-1-harbouring IncI1 plasmids in Escherichia coli with different MLST types in the poultry production pyramid. Front. Microbiol. 5, 519.

Open Access. This is an open-access article distributed under the terms of the Creative Commons Attribution 4.0 International License (https://creativecommons.org/ licenses/by/4.0/), which permits unrestricted use, distribution, and reproduction in any medium, provided the original author and source are credited, a link to the CC License is provided, and changes - if any - are indicated. (SID_1) 